\title{
Preclinically assessed optimal control of postprandial glucose excursions for type 1 patients with diabetes
}

\author{
Thierry Prud'homme, Alain Bock, Grégory François and Denis Gillet
}

\begin{abstract}
Type 1 Patients with Diabetes (Type 1 PwDs) have to frequently adjust their insulin dosage to keep their Blood Glucose concentration (BG) within normal bounds. Meal intakes represent the most important disturbance that has to be accounted for. Its effect differs for every individual as well as for every meal. These specificities are automatically taken into account in the approach proposed in this paper. Model parameters are identified for every couple (Patient, Meal) of interest and optimal control is applied to generate individualized meal specific insulin profiles. The method does not require the use of a continuous BG meter, the profiles being infused in an open-loop manner. Results from a preliminary clinical study are presented. The concept is shown to be effective, despite limitations due to the aggressive execution chosen. Improvements are proposed and a possible pratical implementation is described.
\end{abstract}

\section{INTRODUCTION}

It has been shown that a high average Blood Glucose concentration (BG) over several years increases the risk of severe long term complications, among them nephropathy, retinopathy, cardiovascular diseases or renal failure [1]. On the other hand, too much insulin can lead to a hypoglycemic event, which is a potentially lethal condition.

Type 1 Patients with Diabetes (Type 1 PwDs) have to compensate for events that can be perceived as disturbances from a control point of view. Meal intakes, physical activity [2], stress [3] or menstrual cycles belong to the set of disturbances that have to be accounted for by Type 1 PwDs in their insulin dosage.

Optimized BG control after meal intake has a high potential for improving the treatment of Type 1 PwDs. During the day, the BG depends mainly on the quality of the meal compensations. However, this task is also very challenging because the patient as well as the meal specificities have to be accounted for.

Today, most of the Type 1 PwDs estimate their insulin boluses based on the carbohydrate content of the meal and the measured BG at meal time. They typically infuse this bolus just before the meal intake. One of the main limitations of this strategy lies in the fact that only carbohydrate content is considered, while each meal has its own specificity and can lead to very different BG profiles. Typical BG excursions are shown in Fig. 1. These measurements were taken for the same patient at two consecutive days, at the same

T. Prud'homme is with the Lucerne University of Applied Sciences and Arts, CH 6048 Horw, Switzerland thierry . prudhomme@ hs lu . ch

A. Bock and D. Gillet are with the School of Engineering and G. François is with the Laboratoire d'Automatique, Ecole Polytechnique Fédérale de Lausanne (EPFL), CH 1015 Lausanne, Switzerland alain.bockeepfl.ch
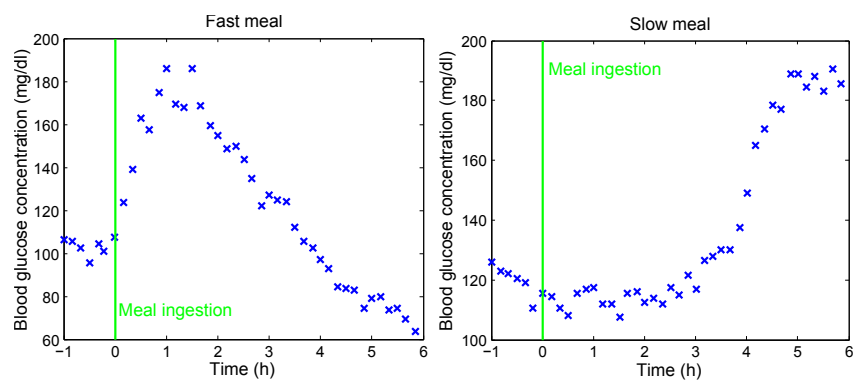

Fig. 1. Postpandrial glucose excursions after a fast and a slow meal for the same patient, meal carbohydrate content, and insulin bolus

daytime, with the same insulin bolus, and with equal meal carbohydrate content but different meal compositions. For the so-called fast meals (mainly carbohydrate content), a high glucose peak is measured approximately 45 minutes after the meal intake. After this peak, the BG decreases very abruptly. Very often, an intervention is needed to compensate for the resulting low BGs. The BG excursion measured for the slow meal (important fat and protein content) is completely different: no peak but a drop followed by a slow and large increase, is observed. BG remains high several hours after the meal intake. This clearly shows that the standard one-shot carbohydrate based insulin bolus cannot handle variations of meal contents, which limits the efficiency of most bolus calculators [4].

The delivery of exogenous insulin has undergone significant changes with the introduction of the insulin pump, a device that allows continuous infusion of fast acting insulin. Nowadays, most pumps are equipped with "smart" meters. These meters have increased computational capacities and can exchange data with insulin pumps. With such devices, the one-shot meal insulin bolus can be replaced by a distributed insulin infusion profile. It makes it possible to shape the profiles and adapt them to the specificity of each meal. This possibility has barely been used so far, and is the topic of this paper.

Today, the research focus is on closed-loop control aiming at a so-called Artificial Pancreas (AP). Two different types of controllers are considered, either a pure feedback controller [5], or model predictive controllers (MPC) [6], [7]. These closed-loop approaches require continuous BG measurements, which are provided by a Continuous Subcutaneous Glucose Monitor (CGM). Such sensors suffer from severe limitations, among them reduced accuracy, potential sensor drop-outs or time delays of approximately 20 minutes. 
Important safety issues related to the automatically changing insulin infusion generated by an AP are another drawback. All these limitations preclude the use of closed-loop controllers, for the time being.

For this reason, an open-loop optimal control strategy is proposed. It does not require the use of a CGM, but it could be extended to use continuous measurements. The strategy provides meal specific distributed insulin profiles. These may be inspected for their safety before the infusion. Patient and meal specific model parameters are identified that are subsequently used to compute optimal insulin infusion profiles. The effectiveness of this method is evaluated in a preliminary clinical study, where BG is monitored and compared to BG excursions shown in Fig. 1, for the same two-meal scenario.

This paper is organized as follows: In section II, the proposed approach is detailed and the model, the parameter identification strategy and the optimal control problem are explained. A possible implementation using existing devices is also sketched in this section. In section III, clinical results are presented and discussed. Finally, in section IV, conclusions are drawn and possible improvements are sketched.

\section{METHODS}

\section{A. Modeling}

The optimal control approach chosen in this work requires a dynamical model of the system. The minimal model, see [8], has been extended to account for a subcutaneous insulin infusion and an oral meal intake. The insulin action and absorption submodel have then been simplified to improve the identifiability of the parameters. The resulting model is given in (1) to (6).

$$
\begin{aligned}
& \frac{d U_{g, g u t}(t)}{d t}=\dot{U}_{g, g u t}(t) \\
& \frac{d \dot{U}_{g, g u t}(t)}{d t}=-2 a_{g} \dot{U}_{g, g u t}(t)-a_{g}^{2} U_{g, g u t}(t)+K_{g} a_{g}^{2} U_{c h o}(t)
\end{aligned}
$$

$$
\begin{aligned}
\frac{d Q(t)}{d t}= & -X(t) Q(t)-S_{g, \text { zero }} Q(t)+U_{\text {endo }} \\
& \quad+\frac{C_{g \rightarrow m m o l}}{M} U_{g, g u t}(t) \\
\frac{d X(t)}{d t}= & -a_{x} X(t)+a_{x} X_{1}(t) \\
\frac{d X_{1}(t)}{d t}= & -a_{x} X_{1}(t)+K_{x} a_{x} \frac{U_{i, s q}(t)}{M} \\
G(t)= & \frac{Q(t)}{V_{g a}}
\end{aligned}
$$

where the states are the gut glucose absorption $U_{g, g u t}$ in $\mathrm{g} / \mathrm{min}$, the time derivative of the gut glucose absorption $\dot{U}_{g, g u t}$ in $\mathrm{g} / \mathrm{min} / \mathrm{min}$, the glucose amount $Q$ in $\mathrm{mmol} / \mathrm{kg}$, the insulin action $X$ in $\min ^{-1}$, and the intermediate insulin action $X_{1}$ in $\min ^{-1}$.

Model parameters are the patient's body weight $M$ in $\mathrm{kg}$, the volume of the accessible compartment per body mass $V_{g a}$ in $\mathrm{L} / \mathrm{kg}$, the unitless bioavailability of the meal of interest $K_{g}$, the inverse of the time constant of the meal of interest $a_{g}$ in $\min ^{-1}$, the insulin sensitivity $K_{x}$ in $\mathrm{kg} / \mathrm{mU}$, the inverse of the time constant of the insulin absorption/action $a_{x}$ in $\min ^{-1}$, the glucose effectiveness at zero insulin $S_{g, \text { zero }}$ in $\mathrm{min}^{-1}$, and the insulin independent endogenous glucose production $U_{\text {endo }}$ in $\mathrm{mmol} / \mathrm{kg} / \mathrm{min}$. $C_{g \rightarrow m m o l}$ converts g of glucose into mmol.

The only manipulated variable is the subcutaneous insulin infusion, $U_{i, s q}(t)$ in $\mathrm{mU} / \mathrm{min}$, and the output is the BG $G(t)$ in $\mathrm{mg} / \mathrm{dL}$. The carbohydrate intake rate $U_{\text {cho }}(t)$ in $\mathrm{g} / \mathrm{min}$ can be viewed as a predictable disturbance.

The model equations (1) to (6) can be expressed with the compact equation $\dot{\boldsymbol{x}}=\boldsymbol{f}(\boldsymbol{x}, u, v, \boldsymbol{\theta})$ where $\boldsymbol{x}$ is the vector of states, $u$ the manipulated input $U_{i, s q}, v$ the predictable disturbance $U_{c h o}$, and $\boldsymbol{\theta}$ represents the vector of model parameters.

Values for $C_{g \rightarrow m m o l}$ and $M$ are known or can be measured directly, while $K_{g}, a_{g}, K_{x}, a_{x}, S_{g, z e r o}$, and $U_{\text {endo }}$ have to be identified. $V_{g a}$ can be assumed constant and equal to the population mean [9]. Therefore, the vector of parameters

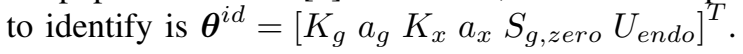

\section{B. Parameter identification}

The model parameters have to be computed for each couple (patient $=P$, meal $=A$ ) as individualized meal specific insulin recommendations are looked for. Therefore, for each meal to be controlled with the proposed approach, corresponding patient BG measurements, with a maximum sampling time of about 1 hour (for example Fig. 5), are needed around the meal time. These measurements can be performed with a classical strip-based meters and do not require the use of a CGM.

These data are exploited to compute the parameter values $\boldsymbol{\theta}_{P, A}^{i d, *}$ for the couple $(P, A)$ that minimize a Maximum A Posteriori (MAP) criterion. The necessary a priori knowledge can be computed using experimental data through a population-based approach, like for example the Iterative Two Stage (ITS) method described by [10], or it can be deduced from available knowledge on the physiology (meal absorption, insulin kinetics). The simple model structure together with the use of a priori knowledge lead to an identifiability with parameters' coefficients of variation below $100 \%$ (cf [11]).

For a MAP identification, illustrated in Fig. 2, the following optimization problem is solved.

$$
\begin{aligned}
& \boldsymbol{M}^{*}=\arg \min J_{i d}(\boldsymbol{M}) \\
& \text { s.t. } J_{i d}(\boldsymbol{M})=\sum_{i=1}^{N_{g}}\left(\frac{\left(\hat{G}\left(t_{i}, \boldsymbol{\theta}_{P, A}^{i d}, v, u\right)-G\left(t_{i}\right)\right)^{2}}{\sigma_{i}^{2}}\right) \\
& \quad+\sum_{j=1}^{N_{\theta}} M_{j}^{2} \\
& \boldsymbol{\theta}_{P, A}^{i d}=\exp (\boldsymbol{A} \boldsymbol{M}+\boldsymbol{B}),
\end{aligned}
$$

where $G\left(t_{i}\right)$ are the $N_{g}$ glucose measurements used for the identification. Measurement errors are supposed to be 


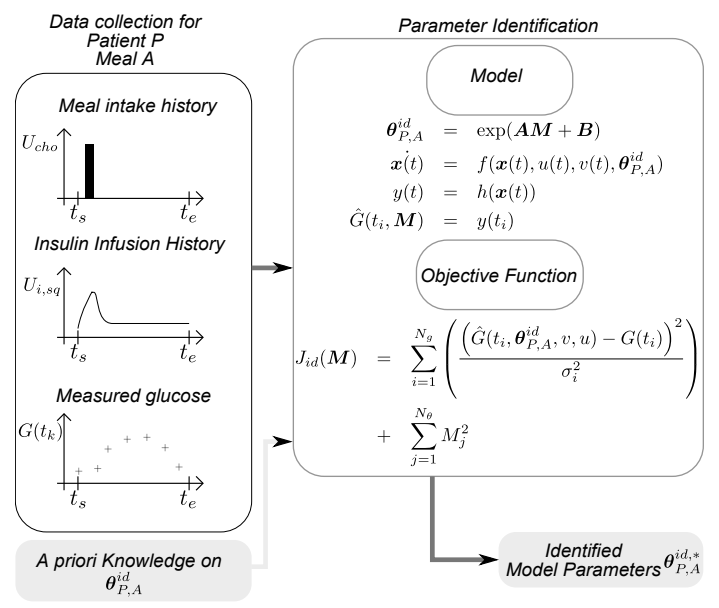

Fig. 2. MAP parameter identification

normally distributed and $\sigma_{i}$ are the corresponding standard deviations. $\hat{G}\left(t_{i}, \boldsymbol{\theta}_{P, A}^{i d}, v, U_{i, s q}\right)$ are model predictions, $N_{\theta}$ is the number of parameters to identify, and $M_{j}$ are the $N_{\theta}$ normalized Gaussian variables described in the Appendix.

\section{Optimal profile generation}

Once the parameters have been identified for a couple ( $\mathrm{P}$, A), a model-based optimal control problem is solved. The manipulated input, i.e. the subcutaneously infused insulin $U_{i, s q}$, is parameterized with a piecewise constant function with constant sampling time $T$. The control horizon is defined by the start time $t_{h, s}$ and the end time $t_{h, e}$.

With such an input parameterization, $U_{i, s q}(t)=\mathcal{U}(\boldsymbol{\pi})$, $\forall t \in\left[t_{h, s}, t_{h, e}\right]$, i.e. $U_{i, s q}(t)$ can be generated using a vector of scalar parameters $\boldsymbol{\pi}=\left[\begin{array}{lll}\pi_{1} & \ldots & \pi_{N_{\pi}}\end{array}\right]^{T}$, which corresponds to a sequence of infusion rates. $N_{\pi}$ is the number of sampling intervals for the period $\left[t_{h, s}, t_{h, e}\right]$. Thus, the optimization problem reads:

$$
\begin{aligned}
& \boldsymbol{\pi}^{*}=\arg \min J_{o p t}(\boldsymbol{\pi}) \\
& \text { s.t. } J_{o p t}(\boldsymbol{\pi})=\int_{t_{h, s}}^{t_{h, e}}\left(\hat{G}\left(t, \boldsymbol{\theta}_{P, A}^{i d, *}, v, \boldsymbol{\pi}\right)-G_{t g, A}(t)\right)^{2} d t \\
& \quad \mathbf{0} \leq \boldsymbol{\pi} \leq \boldsymbol{\pi}_{\max },
\end{aligned}
$$

As seen, the square of the difference between a meal dependent target profile $G_{t g, A}(t)$ for meal $A$ and the predicted blood glucose concentration $\hat{G}(t)$ is minimzed. $\boldsymbol{\pi}_{\max }$ is the insulin pump's maximum infusion rate.

The target profiles for the two meals of interest in the preliminary clinical study are plotted in Fig. 6 .

Patient's BG measurement at time $t_{h, s}$ as well as information on infused insulin before $t_{h, s}$ are used to compute the values of the model states at $t_{h, s}$. Therefore, the computed optimized insulin profile will automatically correct for an initially too low or too high BG. It will also account for the pre-meal insulin infusion history (so called insulin on board), as illustrated in Fig. 3.

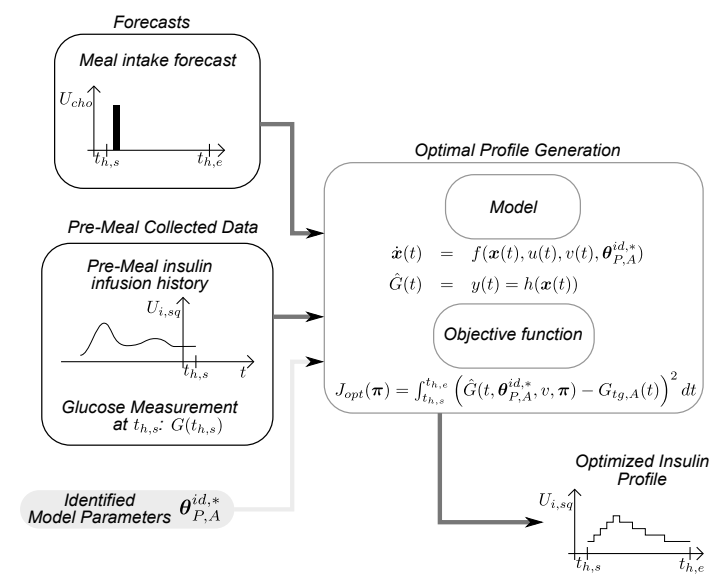

Fig. 3. Overview of the optimization process

\section{Practical implementation}

A possible implementation strategy of this method is sketched in this section.

Today, some insulin pumps are coupled to a "smart" meter. The Accu-Chek ${ }^{\circledR}$ Combo system from Roche is a good example. The glucose meter has been extended and offers additional functionalities. It communicates with the pump and can be used to program a bolus or change the basal rate. On the clinician side, a data management software like AccuChek ${ }^{\circledR} 360^{\circ}$ can be used to collect and process the patient data from the pump and meter. The Accu-Chek ${ }^{\circledR}$ Combo together with the software Accu-Chek ${ }^{\circledR} 360^{\circ}$ is used as an example for the implementation of the method proposed in this article.

The implementation is divided in 4 main steps. They are described in what follows and are illustrated in Fig. 4.

1) Based upon his BG history, the PwD and his Health Care Provider (HCP) identify the meals that have been badly controlled since the last visit. Among these "problematic meals", those who can be potentially improved by the method presented in this paper are selected. The HCP data management software is used to assist the HCP in the selection process. A BG measurement schedule as well as the list of problematic meals are uploaded to the PwD's smart meter.

2) The PwD will follow this BG measurement schedule (e.g. in Fig. 5) for the selected meals. A reminder is implemented in the smart meter to ensure compliance with this schedule. The data collected during theses sequences are stored in the smart meter.

3) During the PwD's next visit to his HCP, model parameters values are identified as shown in II-B for the meals for which the BG measurement schedule has been followed. The computed model parameters are uploaded to the smart meter which is able to perform insulin profile optimization. Typically, the identification strategy is implemented as a module of the HCP data management software. In Fig. 4, it is assumed that the model parameters have been computed for the two 

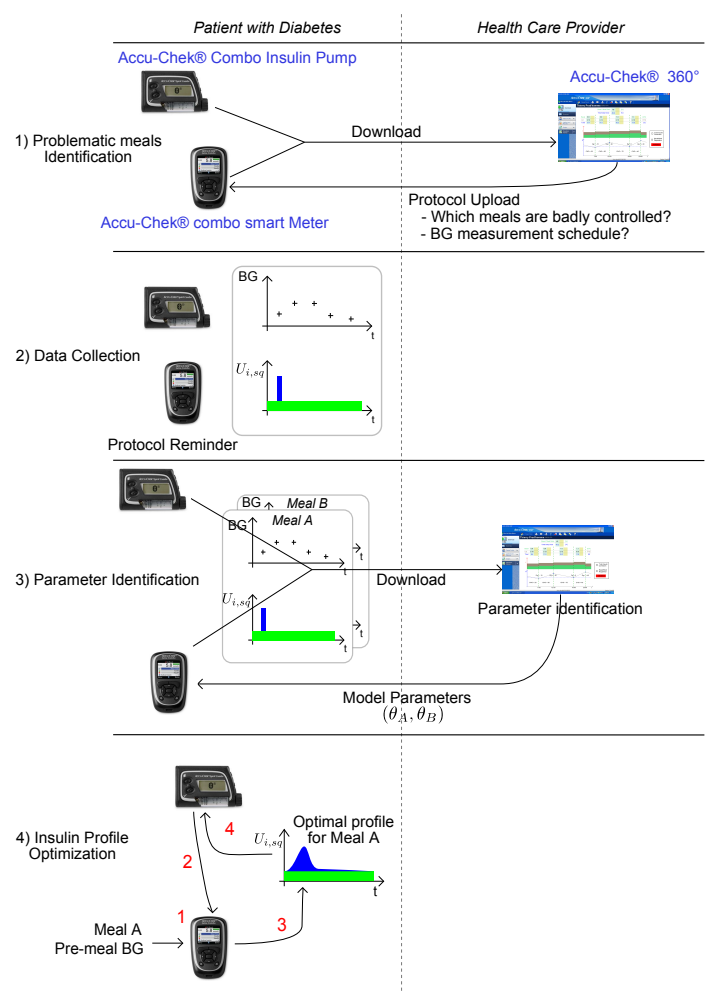

Fig. 4. Possible implementation overview

meals $\mathrm{A}$ and $\mathrm{B}$.

4) The next time one of these two meals is eaten, the PwD's smart meter computes an optimal insulin profile, on the basis of the pre-meal BG, the insulin infusion history, and the identified model parameters for this meal as described in 4. The resulting profile is automatically injected by the insulin pump.

It should be noted that the PwD's safety is of highest importance and needs to be guaranteed before any out-patient implementation.

\section{PRELIMINARY ClinicAl STUdy}

\section{A. Study design}

A clinical study was performed to test the proposed method on 12 Type 1 PwDs. Both fast and slow meals, as depicted in Fig. 1, were considered. For conciseness reasons, only results for 2 patients are discussed.

During the first in-patient period, referred to as "first block", the patients had to eat the two meals on two consecutive days at 9:00 a.m. and to control their BG using the standard therapy. As illustrated in Fig. 5, eight different BG measurements were made around the meal intake with a sampling time of $1 \mathrm{~h}$. The first one was made 1 hour before the meal intake and the last one 7 hours after. The dataset was used to identify the parameters for each couple (P, A) using the strategy discussed in subsection II-B. The a priori knowledge was generated using ITS (cf II-B). $\sigma_{i}$ has been chosen independent of the model and equal to $0.05 G\left(t_{i}\right)$. This describes the assumption that glucose measurements

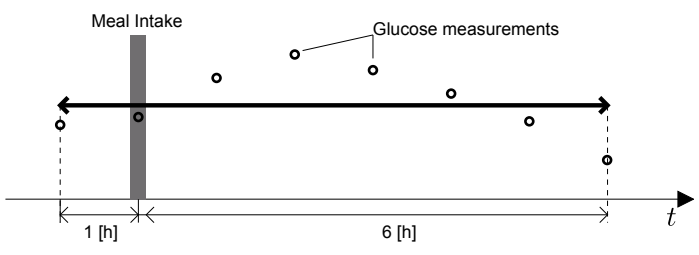

Fig. 5. BG measurement schedule

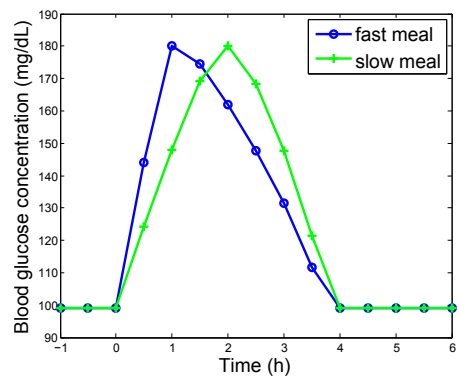

Fig. 6. Glucose target profiles

exhibit a standard deviation equal to $5 \%$ of the measured glucose value. This value is of particular importance when the number of measurements is low and the weight of the Bayesian term in the objective function is not negligible.

The second in-patient period, referred to as "second block", took place approximately 2 months after the first one. During this period, the optimization routine described in subsection II-C was used to compute optimized insulin profiles. $t_{h, s}$ was set to 1 hour before meal intake and $t_{h, e}$ to 6 hours after meal intake. A sampling period $T$ of 10 min was fixed. This led to $N_{\pi}=42$ decision variables to optimize. The two target profiles depicted in Fig. 6 were used for the fast and slow meals. It can be noted that these target profiles exhibit similar shapes, but the peak of the profile used for the slow meal is shifted by an hour. The resulting optimized insulin profiles were infused to the patients, while their BG was measured every $10 \mathrm{~min}$ from intravenous blood draws to assess the performances of the proposed approach.

\section{B. Study results}

The results of the study for the two patients are shown in Fig. 7 to 10. On the left hand side, the infused insulin profiles for the two blocks are plotted. The green and the blue curves represent the one-shot boluses and the optimal insulin profiles, respectively. On the right hand side, the BG excursions measured in the two blocks are plotted. The green and blue lines represent the measured BGs in the first and second block, respectively. The dotted blue line gives the expected BG excursion for the second block. The prediction capabilities of the model can be studied by comparing the plain blue line with the dotted blue line.

Similar conclusions can be drawn for the two patients. At the beginning of the optimization horizon (before meal intake), the optimization computed large insulin amounts. It can be seen in the four figures that these corrections were efficient. For the fast meals, this large initial amount 

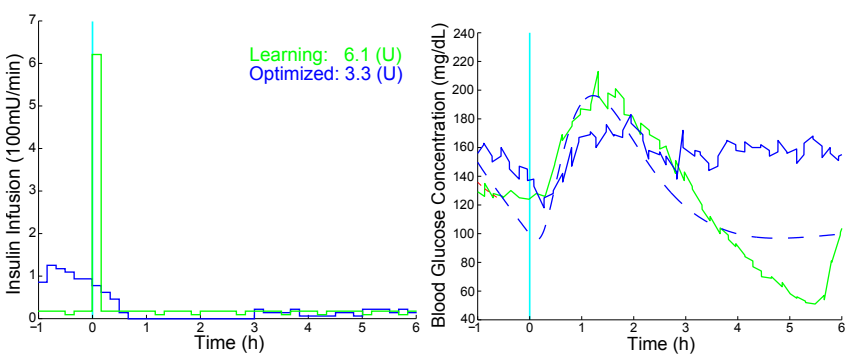

Fig. 7. Clinical results, patient 1, fast meal
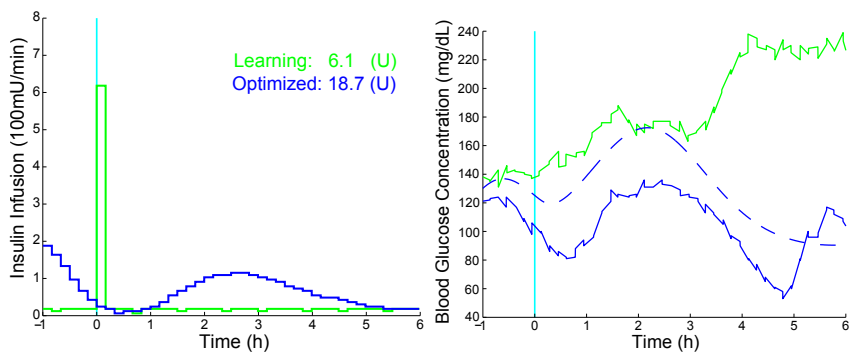

Fig. 8. Clinical results, patient 1 , slow meal

also compensates for the effect of the meal, as nearly no additional insulin injection was needed after meal intake. For the slow meals, a second insulin injection was necessary between 1 and 4 hours after the meal intakes. The shapes of the BG predictions and measurements were similar, for the fast as well as for the slow meals.

Table I gives the values of the objective function $J_{o p t}$ for the two blocks. Except for the couple (patient 1, fast meal), the optimized insulin profiles led to significant reductions of $J_{\text {opt }}$. These reductions are particularly important for the slow meals. The insulin infused between 1 hour and 4 hours after the intake of the slow meals prevents from the hyperglycemia that was observed during the first blocks.

For the couple (patient 1, fast meal), the BG excursion measured at the first block was close to the target profile except for a hypoglycemic event that occurred 4-5 hours after the meal intake. To avoid this, the optimization recommended a smaller insulin infusion. However, this correction was too important and led to a higher value for $J_{o p t}$.

As already mentioned, the BG model predictions for the second block (plain blue line) exhibit shapes in accordance
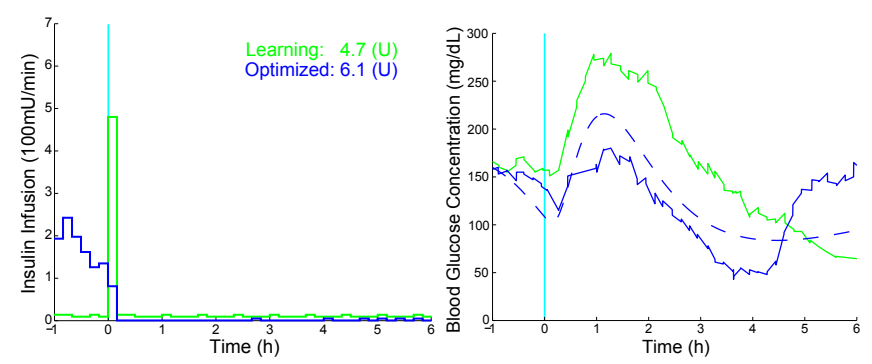

Fig. 9. Clinical results, patient 2, fast meal
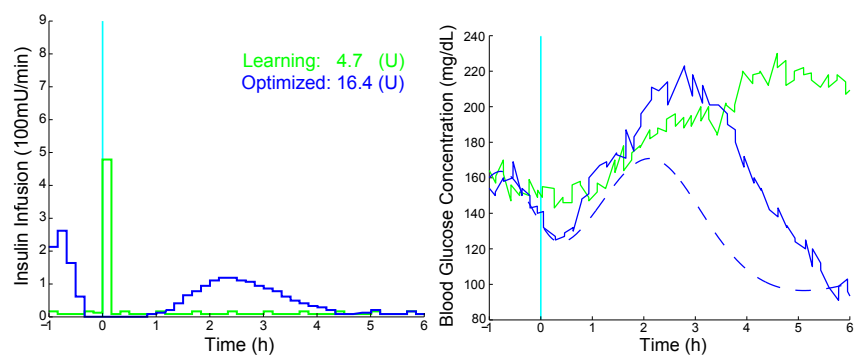

Fig. 10. Clinical results, patient 2, slow meal

TABLE I

VALUES OF $J_{\text {opt }}$ FOR THE $1^{\text {st }}$ AND $2^{\text {nd }}$ BLOCK AND THEIR RELATIVE VARIATION. BG MEASUREMENTS AFTER HYPOGLYCEMIA EVENTS WERE NOT TAKEN INTO ACCOUNT FOR THE COMPUTATION OF $J_{\text {opt }}$.

\begin{tabular}{|l|c|c|c|}
\hline & $\begin{array}{c}\text { Standard } \\
1^{\text {st }} \text { block }\end{array}$ & $\begin{array}{c}\text { Optimized } \\
2^{\text {nd }} \text { block }\end{array}$ & $\begin{array}{c}\text { Relative } \\
\text { variation }\end{array}$ \\
\hline Patient 1 fast meal & 4776 & 7921 & $+65.89 \%$ \\
Patient 1 slow meal & 19323 & 6000 & $-69.95 \%$ \\
Patient 2 fast meal & 24440 & 12143 & $-50.31 \%$ \\
Patient 2 slow meal & 36874 & 10858 & $-70.55 \%$ \\
\hline
\end{tabular}

with the expected BG excursions (dotted blue line). However, it should also be noted that the optimization procedure led to too aggressive corrections between the 2 blocks. The size of the different boluses can be found in Table II. As seen, they differ significantly.

Obviously, the model is not able to predict BG with a good confidence when the insulin profile used for the identification differs too much from the insulin profile used for the computation of the predictions. The limitations of the predictions capabilities of the model can have different reasons, some of them are listed below:

- The choice of the model structure was mainly driven by the identifiability of the parameters and by the accuracy of the fits. It is known that glucagon, free fatty acids or growth hormones, as well as many other molecules, influence the BG. They have been intentionally neglected, as it would have been impossible to quantify their contribution with only a few BG measurements. Therefore, the model used for this implementation is an oversimplification of the glucose metabolism. This is the main reason for the limited prediction capabilities.

- The model parameters for a couple (P,A) were identified on a single dataset collected during the first block. As mentioned earlier, several disturbances like stress, physical activity or menstrual cycles have not been considered. These disturbances should, at least, be filtered

TABLE II

TOTAL INFUSED INSULIN (U)

\begin{tabular}{|l|c|c|}
\hline & Standard & Optimized \\
\hline Patient 1 fast meal & 6.1 & 3.3 \\
Patient 1 slow meal & 6.1 & 18.7 \\
Patient 2 fast meal & 4.7 & 6.1 \\
Patient 2 slow meal & 4.7 & 16.4 \\
\hline
\end{tabular}


to minimize their influence on the model parameters identified. This could be done, e.g., by using more than one dataset to identify the parameters for a couple (P,A).

- Insulin absorption exhibits nonlinearities with respect to the amount of injected insulin. However, the model used in this work is linear. Therefore, the identified parameters should not be used to compute predictions for insulin infusion profiles that differ too much from the profiles used for the identification.

- Physiological parameters of a PwD change over time. As the second block was performed approximately 2 months after the first one, the identified model parameters may not be accurate enough anymore.

\section{CONCLUSION AND OUTLOOK}

The results presented in this paper are promising. An optimized distributed insulin profile led to a reduced BG peak after a fast meal intake. It also corrected for the hyperglycemic events observed during the first block a few hours after the slow meal intakes. However, corrections were shown to be potentially aggressive and future work should include the following improvements:

- Small step strategy: The difference between two consecutive insulin profiles for the same meal should be penalized in $J_{o p t}$, as proposed in [12]. Indeed, the preliminary clinical results have shown that the extrapolation capabilities of the model are too limited to allow big variations between two consecutive insulin profiles. By introducing this penalty term in $J_{o p t}$, several iterations will be necessary to converge to an optimal insulin profile for a couple (P, A), but safety will be improved.

- No meal-dependent target profile: The proposed approach requires meal-dependent target $\mathrm{BG}$ profiles as shown in Fig. 6. It assumes that some a priori knowledge is available on the absorption dynamics of each meal. In practice, this is very difficult to conceive. Instead, a constant target profile could be used for all of the meals and a new objective function $J_{o p t}$ should be used. This new $J_{o p t}$ would penalize deviation from target in an asymmetrical manner. BGs below target would have a much stronger contribution to $J_{o p t}$ than BGs above target to avoid hypoglycemia. An objective function ins red by the metric defined in [13] could be used advantageously.

- Robust identification: Some of the model parameters ( $K_{x}, a_{x}, S_{g, z e r o}$, and $U_{\text {endo }}$ ) do not depend on the meal but only on the patient. In this paper, all the parameters are identified for each couple ( $\mathrm{P}, \mathrm{A})$. Results could be improved if the patient dependent parameters would be identified using datasets from different meals.

- Learning horizon reduction: The learning horizon should be reduced to $4 \mathrm{~h}$ for practical reasons. Indeed,the time between two consecutive meal intakes is frequently smaller than $6 \mathrm{~h}$.

However, the main limitation of the proposed approach lies in the simplicity of the model used for optimization. This is clearly an advantage for identification, which is made possible on the basis of sole glucose measurements, but meanwhile it can be a drawback at the optimization stage. To be able to predict the real optimal insulin profiles for a given patient, the tendency model we are using should fulfill some adequacy requirements [14]. In practice, these requirements are hard to meet, but future research should compare the accuracy of this simplified model with more complex models (for example [9], [15]). Also, to avoid hyper- or hypoglycemia, robust optimization (i.e. optimization considering uncertainty, e.g. deduced from confidence intervals) should be investigated.

\section{REFERENCES}

[1] DCCT, "The effect of intensive treatment of diabetes on the development and progression of long-term complications in insulin-dependent diabetes mellitus." New Engl J Med, vol. 329, pp. 977-986, 1993.

[2] B. Zinman, N. Ruderman, B. N. Campaigne, J. T. Devlin, and S. H. Schneider, "Physical Activity/Exercise and Diabetes," Diabetes Care, vol. 27, no. supplement 1, pp. S59-S62, 2004.

[3] D. A. Finan, H. Zisser, L. Jovanovic, W. C. Bevier, and D. E. Seborg, "Automatic Detection of Stress States in Type 1 Diabetes Subjects in Ambulatory Conditions," Ind Eng Chem Res, vol. 49, pp. 7843-7848, 2010.

[4] O. K. Hejlesen, S. Andreassen, R. Hovorka, and D. A. Cavan, "DIASthe diabetes advisory system: an outline of the system and the evaluation results obtained so far," Computer methods and programs in biomedicine, vol. 54, no. 1-2, pp. 49-58, Sept. 1997.

[5] S. A. Weinzimer, G. M. Steil, K. L. Swan, J. Dziura, N. Kurtz, and W. V. Tamborlane, "Fully automated closed-loop insulin delivery versus semiautomated hybrid control in pediatric patients with type 1 diabetes using an artificial pancreas," Diabetes Care, vol. 31, no. 5, pp. 934-939, 2008.

[6] R. Hovorka, V. Canonico, L. J. Chassin, U. Haueter, M. MassiBenedetti, M. O. Federici, T. R. Pieber, H. C. Schaller, L. Schaupp, T. Vering, and M. E. Wilinska, "Nonlinear model predictive control of glucose concentration in subjects with type 1 diabetes," Physiological Measurement, vol. 25, no. 4, p. 905, 2004.

[7] P. Dua, F. Doyle, and E. Pistikopoulos, "Model-based blood glucose control for type 1 diabetes via parametric programming," IEEE Trans Biomed Eng, vol. 53, no. 8, pp. 1478 -1491, aug. 2006.

[8] R. N. Bergman, Y. Z. Ider, C. R. Bowden, and C. Cobelli, "Quantitative estimation of insulin sensitivity," Am J Physiol Gastrointest Liver Physiol, vol. 236, no. 6, pp. G667-677, 1979.

[9] R. Hovorka, F. Shojaee-Moradie, P. V. Carroll, L. J. Chassin, I. J. Gowrie, N. C. Jackson, R. S. Tudor, A. M. Umpleby, and R. H. Jones, "Partitioning glucose distribution/transport, disposal, and endogenous production during IVGTT." American journal of physiology. Endocrinology and metabolism, vol. 282, no. 5, pp. E992-1007, 2002.

[10] P. Vicini and C. Cobelli, "The iterative two-stage population approach to ivgtt minimal modeling: improved precision with reduced sampling," Am J Physiol-Endoc M, vol. 280, no. 1, pp. E179 -E186, January 2001.

[11] M. E. Wilinska, L. J. Chassin, H. C. Schaller, L. Schaupp, T. R. Pieber, and R. Hovorka, "Insulin kinetics in type-1 diabetes: continuous and bolus delivery of rapid acting insulin," IEEE Transactions on Biomedical Engineering, vol. 52, no. 1, pp. 3-12, 2005.

[12] T. Prud'homme and C. Cris, "US2010/0125241 A1. Prandial blood glucose excursion optimization method via computation of timevarying optimal insulin profiles and system thereof," 2008.

[13] B. P. Kovatchev, W. L. Clarke, M. Breton, K. Brayman, and A. Mccall, "Quantifying temporal glucose variability in diabetes via continuous glucose monitoring: Mathematical methods and clinical application." Diabetes Technol Ther, vol. 7, no. 6, pp. 849-862, 2005.

[14] J. Forbes, T. Marlin, and J. MacGregor, "Model adequacy requirements for optimizing plant operations," Computers \& Chemical Engineering, vol. 18, no. 6, pp. 497-510, 1994.

[15] C. Dalla Man, R. A. Rizza, and C. Cobelli, "Meal simulation model of the glucose-insulin system," IEEE Trans Biomed Eng, vol. 54, no. 10, pp. 1740-1749, 2007. 


\section{APPENDIX}

A. Maximum A Posteriori identification strategy: Transformation

In the Maximum A Posteriori method, the vector $\boldsymbol{\theta}$ of model parameters is a random variable. In this paper, this random variable is assumed to have a log-normal distribution. This distribution is widely used in the context of physiological model parameters as it ensures that any realization of this random variable will have a positive value.

By definition we have:

$$
\theta_{i}=\exp (\boldsymbol{\Theta})_{i} \text { for each } i \in\left\{1, \cdots, N_{\theta}\right\},
$$

with $N_{\theta}=\operatorname{dim}(\boldsymbol{\theta})$, and $\boldsymbol{\Theta}=\left[\Theta_{1}, \cdots, \Theta_{N_{\theta}}\right]$ is a random vector having a multivariate normal distribution. $\Theta$ is thus fully defined by a mean vector $\mu_{\Theta}$ and a covariance matrix $\Sigma_{\Theta}$ with:

$$
\begin{aligned}
\boldsymbol{\mu}_{\boldsymbol{\Theta}} & =E[\boldsymbol{\Theta}] \\
\boldsymbol{\Sigma}_{\boldsymbol{\Theta}} & =E\left[(\boldsymbol{\Theta}-E[\boldsymbol{\Theta}])(\boldsymbol{\Theta}-E[\boldsymbol{\Theta}])^{T}\right],
\end{aligned}
$$

with the autocorrelation coefficients being:

$$
\sigma_{\Theta}(i)=\sqrt{\Sigma_{\Theta}(i, i)},
$$

and the correlation coefficients:

$$
\sigma_{\Theta}(i, j)=\frac{\Sigma_{\Theta}(i, j)}{\sigma_{\Theta_{i}} \sigma_{\Theta_{j}}} .
$$

A transformation that defines a one-to-one relationship between $\Theta$ and a vector $M$ of uncorrelated normalized (zero mean and identity covariance) Gaussian vector is applied. $\boldsymbol{M}$ is also of dimension $N_{\theta}$.

This transformation is defined as follows:

$$
\Theta=A M+B
$$

where $\boldsymbol{A}$ is called the transformation matrix.

$\boldsymbol{A}$ and $\boldsymbol{B}$ should be uniquely defined by $\boldsymbol{\mu}_{\Theta}$ and $\boldsymbol{\Sigma}_{\Theta}$. To determine the relationship between these three entities, first the mean of $\Theta$ is computed as follows:

$$
E[\boldsymbol{\Theta}]=\boldsymbol{A} E[\boldsymbol{M}]+\boldsymbol{B} .
$$

$M$ has zero mean. Thus:

$$
E[\Theta]=\boldsymbol{\mu}_{\Theta}=\boldsymbol{B} .
$$

The covariance of $\Theta$ is computed:

$$
\boldsymbol{\Sigma}_{\boldsymbol{\Theta}}=E\left[(\boldsymbol{\Theta}-E[\boldsymbol{\Theta}])(\boldsymbol{\Theta}-E[\boldsymbol{\Theta}])^{T}\right] .
$$

$\boldsymbol{\Theta}=\boldsymbol{A} \boldsymbol{M}+\boldsymbol{B}$ and $E[\boldsymbol{\Theta}]=\boldsymbol{B}$, thus:

$$
\boldsymbol{\Sigma}_{\boldsymbol{\Theta}}=E\left[(\boldsymbol{A} \boldsymbol{M})(\boldsymbol{A} \boldsymbol{M})^{T}\right] .
$$

Developing:

$$
\boldsymbol{\Sigma}_{\Theta}=E\left[\boldsymbol{A} \boldsymbol{M} \boldsymbol{M}^{T} \boldsymbol{A}^{T}\right]=\boldsymbol{A} E\left[\boldsymbol{M} \boldsymbol{M}^{T}\right] \boldsymbol{A}^{T} .
$$

However $E\left[M M^{T}\right]$ is the identity matrix, remembering that $M$ is a set of independent normalized Gaussian variables. Thus, we have:

$$
\boldsymbol{\Sigma}_{\Theta}=\boldsymbol{A} \boldsymbol{A}^{T}
$$

If $\boldsymbol{A}$ is chosen as a triangular matrix, it can easily be proven that the components $A(i, j)$ of the matrix $\boldsymbol{A}$ are defined by the two following equations:

$$
\begin{aligned}
& A(j, j)=\sqrt{\sigma_{\Theta}(j)^{2}-\sum_{k=1}^{j-1} A(j, k)^{2}} \\
& A(i, j)=\frac{\sigma_{\Theta}(j) \sigma_{\Theta}(i) \sigma_{\Theta}(j, i)-\sum_{k=1}^{j-1}(A(j, k) A(i, k))}{A(j, j)} .
\end{aligned}
$$

$\boldsymbol{A}$ and $\boldsymbol{B}$ are now fully defined. As a reminder, we have:

$$
\boldsymbol{\theta}=\exp (\boldsymbol{\Theta})=\exp (\boldsymbol{A} \boldsymbol{M}+\boldsymbol{B}) .
$$

B. Maximum A Posteriori identification strategy: Objective function

The minimized objective function for the parameter identification takes the following form:

$$
J_{i d}=\sum_{i=1}^{N_{g}}\left(\frac{\left(\hat{G}\left(t_{i}, \boldsymbol{M}\right)-G\left(t_{i}\right)\right)^{2}}{\sigma_{i}^{2}}\right)+\sum_{j=1}^{N_{\theta}} M_{j}^{2},
$$

where $N_{g}$ is the total number of glucose measurements used for the identification and $N_{\theta}$ is the number of parameters being identified. $M_{j}$ are the corresponding $N_{\theta}$ normalized Gaussian variables which together are the previously mentioned $M$ vector. 